\title{
Elucidation of the softening mechanism of fabric softeners
}

Keywords: fabric softener, mechanism, hydrogen bonding, non-frozen bound water, textile, cotton

\section{Introduction}

Research in the early 1930s showed that cationic surfactants, which were developed to improve the water-fastness of direct dyes on cellulose, also worked as softening agents. ${ }^{1}$ Cationic fabric softeners for consumer use were first introduced to the US market around $1955 .^{2}$ Although the initial growth was not high, the global market has increased remarkably. Nowadays, the fabric softener is regarded as an essential item in each household. In the current market, there exist various types of fabric softeners with different functions such as fragrance, deodorant, and antibacterial agent. The basic purpose of the fabric softener, however, has not changed. When we touch soft laundered fabrics, the comfortable feeling and sensation makes our daily life more satisfying.

Thus, the fabric softener is considered to be a very convenient product that easily and effectively adds a comfortable feel to clothes and towels, especially in the case of fabrics made of cotton. A wellknown and conventional theory of the softening mechanism states that the softening effect results from the "decrease of friction" giving us the image of increase in "sliding" between fibers and yarns. ${ }^{1,3}$ The softening agent is made of a cationic surfactant with two long alkyl chains. In this theory, these alkyl chains are adsorbed on the fiber surface so that this surface is covered by a highly hydrophobic alkyl chain layer oriented towards the air. This condition causes a decrease in friction between the fibers, resulting in the softening of yarns in cloths. There have been many reports of research based on this friction-reduction theory to try to understand the relationship between friction-related textile properties and their softening effect. For example, Larrat ${ }^{4}$ reported that there was no relationship between friction measured by a Handle-o-meter and the softness level; however, a decrease in friction was observed when a friction meter was used instead. On the other hand, Roeder ${ }^{5}$ and Olofsson ${ }^{6,7}$ reported that an important relationship exists between the friction between fibers and the softness of the fibers; as a result, the relationship between the static and dynamic coefficients of friction is regarded as a very important one, and a low value of the static coefficient of friction is believed to be essential for the softening effect. Motoyama et al. ${ }^{8}$ maintained that friction is surely related to the softness level, but an absolute value of the softness and its variation trend depend on how it is measured; thus, a softening agent that can decrease friction between fibers does not always decrease friction between fibers and human skin. Finally, the results of Crutzen ${ }^{9}$ indicate that an agent with a high softening effect does not always effectively reduce the frictional coefficient. While all of these reports are very interesting, there has been no direct research on the basic principle of the softening phenomenon, which is the motivation for our current research.

Most consumers can relate to what happens when wet cotton rags are forgotten and left to dry naturally-the dry rags are so hard that they feel as if they have been glued together. A fabric softener does an excellent job on reducing this hardness. Thus, we focused on this commonly observed hardening phenomenon and investigated how
Volume 2 Issue 2 - 2017

\author{
Takako Igarashi, Koichi Nakamura \\ Global R\&D-Household Products Research, Kao Corporation, \\ Japan
}

Correspondence: Takako Igarashi, Global R\&D-Household Products Research, Kao Corporation, 1334, Minato Wakayama city, Wakayama 640-8580, Japan, Tel 81734265019 , Fax 81734265032, Email igarashi.takako@kao.co.jp

Received: May 18, 2017 | Published: June 21, 2017

fabric softeners work. In the course of our research, we discovered the following: there is a characteristic pattern of change in the hardness of cotton yarns upon the application of a mechanical force such as bending by the Kawabata evaluation system (KES); naturally dried cotton yarns become very soft and fluffy when they are completely dried in a vacuum. These two discoveries indicate that the hardening and softening of yarns take place through the following mechanisms. The main hardening factor of cotton is the cross-linkage of hydrogen bonds mainly in non-frozen bound water ${ }^{10}$ between fibers; these bonds can be broken by mechanical force, as observed in KES bending tests. The softening effect of cotton occurs by inhibiting cross-linkage of these hydrogen bonds as shown in Figure. ${ }^{11}$

On the other hand, when we tried to investigate the relationship between the condition of macro states of softener adsorption and its effect, we found that softening agents have the unexpected tendency to favor adsorption on the outer parts of cloths and yarns. These softening agents show low probability of existence at cross-points between yarns and in the inner part of yarns. These results are in good agreement with those reported by Sakai et al. ${ }^{12}$ who studied the mechanism of softener adsorption on the surface of a mica plate, which was found to be driven by collision of the softener vesicle, and the adsorbed softener remained at the same place without any migration. The authors stated that the main driving force was not considered to be based on electrostatic attraction.

We also came across a very interesting phenomenon when we compared the softness of cloths with and without a softening agent at the cross-points. Contrary to our expectation, we found that the cloth whose cross points had less softening agent was softer and had a higher volume than the cloth whose cross points were covered by a softening agent. This result indicates that the role of softener adsorbed on the external part of yarns or cloth is more important than that of softener at the cross-points where friction exists between fibers or yarns.

A softening agent imparts high hydrophobicity to the part of fibers where it is adsorbed. It plays two important roles: First, it decreases the meniscus force-an attractive force-between fibers in the drying process, thus preventing any decrease in distance between the fibers. Second, the softening agent inhibits the cross-linkage between 
hydrogen bonds made of bound-water between the fibers. On the other hand, as the softener is unlikely to be present in the inner part of the yarn, cross-linkage of hydrogen bonds inevitably occurs and a hardened part is formed, like the duramen (or heartwood) of a tree.
Thus, the two different physical properties of the soft outer part and the hard inner duramen are the key factors that result in the soft and bouncy feel of fabric laundered with a softener as shown in Figure 2. ${ }^{13}$

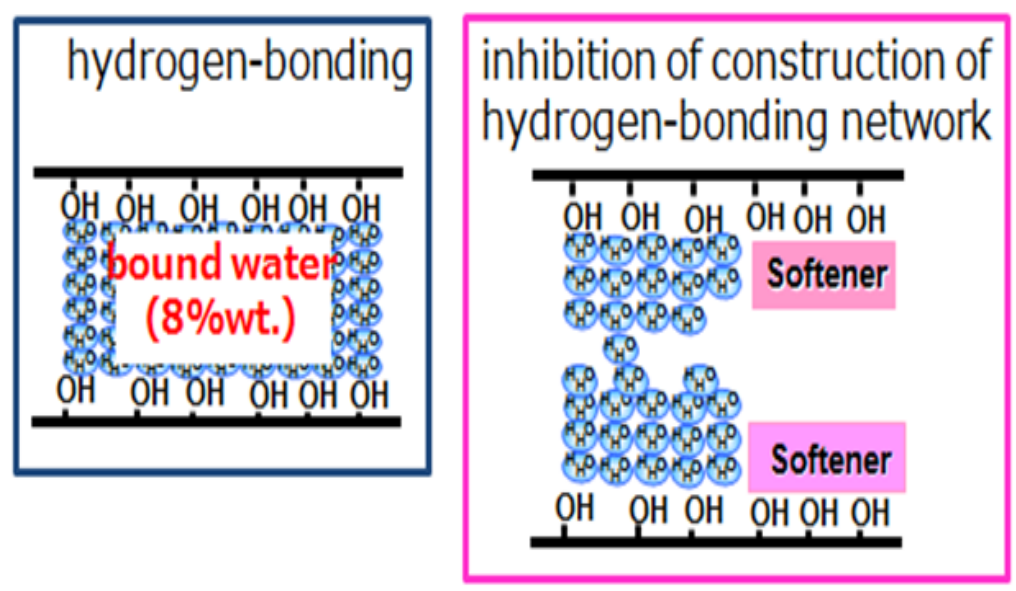

Figure I Important role of softening agent.

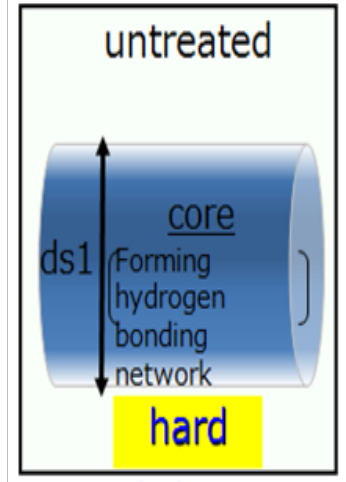

whole yarn

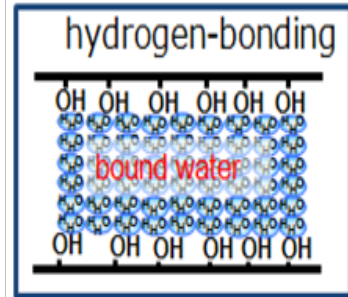

Figure $\mathbf{2}$ The merit of gradation of softening agent in the yarns.

\section{Conclusion}

We hope that our findings on the softening mechanism will inspire new ideas for developing better softening agents and new textile manufacturing technology that will meet the demands of consumers. We also hope to contribute to the realization of a more comfortable and satisfying lifestyle for consumers through these works.

\section{Acknowledgments}

None.

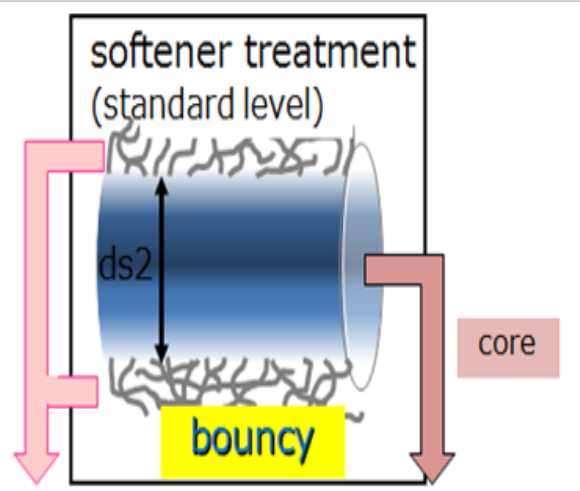

outer part of yarn inner part of yarn
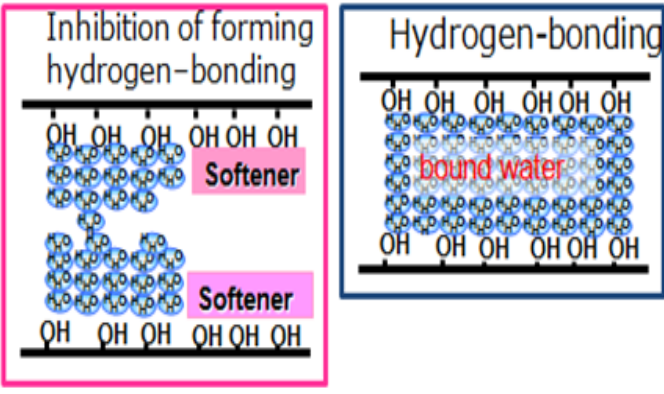

\section{Conflict of interest}

Author declares there is no conflict of interest in publishing the article.

\section{References}

1. Evans WP. Cationic fabric softeners. Chem Ind. 1969;5:893-903.

2. Egan RR. Cationic surface active agents as fabric softeners. $J$ Am Oil Chem Soc. 1978;55(1):118-121. 
3. Miyasaka H. Recent Trends in development of domestic fabric softeners. Oleoscience. 2005;5(10):463-471.

4. Larrat E. An inquiry into the influence of application conditions on softener efficiency. Am Dyest Rep. 1966;55:30.

5. Roeder HL. Measurements of the influence of finishing agents on the friction of fibers. $J$ Textile Inst Trans. 1953;44(6):247-265.

6. Olofsson B, Gralen N. Measurement of friction between single fibers. Text Res J. 1947;17(9):488-496.

7. Olofsson B, Gralén N. Measurement of friction between single fibers frictional properties of viscose rayon staple fibers. Text Res J. 1950;20(7):467-476.

8. Motoyama R, Saiuchi T. Fabric softeners. Textile. 1961;13:518-521.

9. Crutzen AM. Study of the dittalowdimethyl ammonium chloride interaction with cellulose. J Am Oil Chem Soc. 1995;72(1):137-143.
10. Nakamura K, Hatakeyama T, Hatakeyama H. Studies on bond water of cellulose by differential scanning calorimetry. Text Res J. 1981;51:607613.

11. Igarashi T, Morita N, Okamoto Y, et al. Elucidation of softening mechanism in rinse cycle fabric softeners. Part 1: Hydrogen bonding. J Surfactants Deterg. 2016;19:183-192.

12. Sakai T, Matsumoto K, Suzuki K, et al. Dynamic observation of vesicle fusion on mica substrate in water. International Association of Colloid and Inter surface Scientists. 2012.

13. Igarashi T, Nakamura K, Hoshi M, et al. Elucidation of softening mechanism in rinse cycle fabric softeners. Part 2: Uneven adsorption-the key phenomenon to the effect of fabric softeners. J Surfactants Deterg. 2016;19:759-773. 